\title{
UNBIASED MULTI-PARAMETRIC ESTIMATIONS OF DISTANCES AND PECULIAR VELOCITIES OF THE GALAXIES
}

\author{
T.B. GEORGIEV \\ Rozhen National Astronomical Observatory \\ BG-4700 Smolyan \\ Bulgaria
}

Accurate estimates of distances and peculiar velocities for galaxies may be derived with the help of multi-dimensional regression analysis using two or more distance indicators $p_{\mathbf{k}}$ (corrected apparent sizes, luminosities, other distance-dependent quantities) and some calibrators $q_{1}$ (velocity dispersions of the ellipticals or HI-line widths of the spirals, mean surface brightness, colours, other distance-independent quantities) together, cf. Georgiev (1992). Here an example is given for 349 spiral galaxies with axial ratio $D / d>1.4$ from the sample of Fisher \& Tully (1981). The $p$-values are the major axis and the blue magnitude and the $q$-values are the HI line width, type and axial ratio.

The deceleration laws in the nearby universe are $\log p_{k}=-\log V+$ const $_{k}$, where using the Hubble law $\log V$ changes $\log R$ (Fig. 1). The deviations $\Delta \log V_{\text {ik }}=\Delta \log p_{\text {ik }}=\log V_{\mathrm{i}}+\log p_{\text {ik }}-$ const $_{\mathrm{k}}$ are unbiased raw estimations of the peculiar velocities. The regressions $\Delta \log p_{\mathrm{k}}=\mathrm{f}_{\mathrm{k}}\left(q_{\mathrm{t}}\right)$ (Figs. 2 - 4) give better estimations $\left\langle\log V_{\mathrm{ir}}\right\rangle=-\log p_{\mathrm{ik}}+$ const $_{\mathrm{k}}-\mathrm{f}_{\mathrm{k}}\left(q_{\mathrm{il}}\right)$. Figure 2 presents the shifted Tully-Fisher (TF) diagram and Fig. 5 shows the deceleration diagram after TF-corrections of the major axes.

The regressions $\Delta \log p_{\mathrm{k}}=\mathrm{F}_{\mathrm{k}}\left(q_{1}, q_{2}, \ldots\right)$ are the multi-parametric generalizations of the TF (or Faber-Jackson) relations. The initial velocity estimations in the multi-dimensional method are $\left.<\log V_{\mathrm{ir}}\right\rangle=-\log p_{\mathrm{ik}}+$ const $_{\mathrm{k}}-\mathrm{F}_{\mathrm{k}}\left(q_{\mathrm{i} 1}, q_{\mathrm{i} 2}, \ldots\right)$ and the final estimations $\left\langle\log V_{\mathrm{i}}>\right.$ are obtained by the linear regression $\log V=\mathrm{G}\left(\left\langle\log V_{1}\right\rangle,\left\langle\log V_{2}\right\rangle, \ldots\right)$. The mean-square value of the final peculiar velocity estimations $\delta \log V_{\mathrm{i}}=\log V_{\mathrm{i}}-\left\langle\log V_{\mathrm{i}}>\right.$ occurs about 1.2 times lower than that obtained by the pure TF-method (Fig. 6).

The general multi-dimensional method is performed by one C-program of the author including the graphics library of Dr. L. Georgiev (1991).

\section{References}

Fisher, J.R. \& Tully, R.B., 1981. Astrophys. J. Suppl., 47, 139.

Georgiev, L., 1991. Private communication.

Georgiev, Ts., 1992. Sov. Astron. Let. 18 (Pis'ma v Astron. Zh., 18, 739).

715

H. T. MacGillivray et al. (eds.), Astronomy from Wide-Field Imaging, 715-717.

C) 1994 IAU. Printed in the Netherlands. 


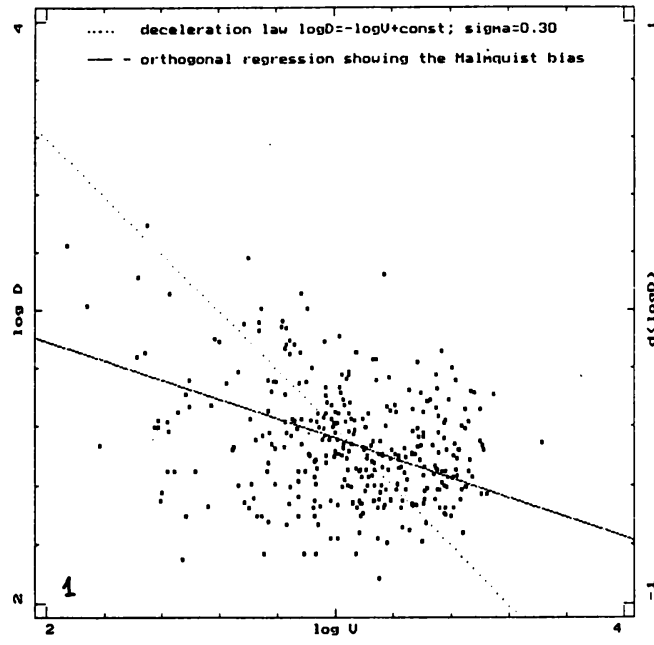

Figure 1. The observed deceleration diagram where $\mathrm{V}$ is the corrected Hubble velocity and D is the corrected apparent major axis: 1 - the deceleration law $\log \mathrm{D}=-\log \mathrm{V}+$ const; sigma $=0.30 ; 2$ - the orthogonal regression showing the Malmquist bias.

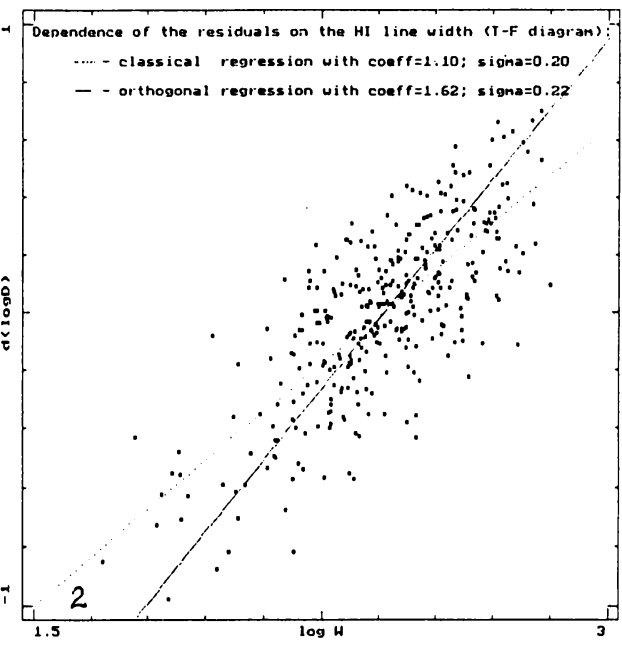

Figure 2. Shifted TF-diagram where d(logD) is the deviation from the deceleration law given in Fig. 1: 1 - classical regression; coef. = 1.10; sigma $=0.20 ; 2$ - orthogonal regression; coef. $=1.62$; sigma $=0.22$.
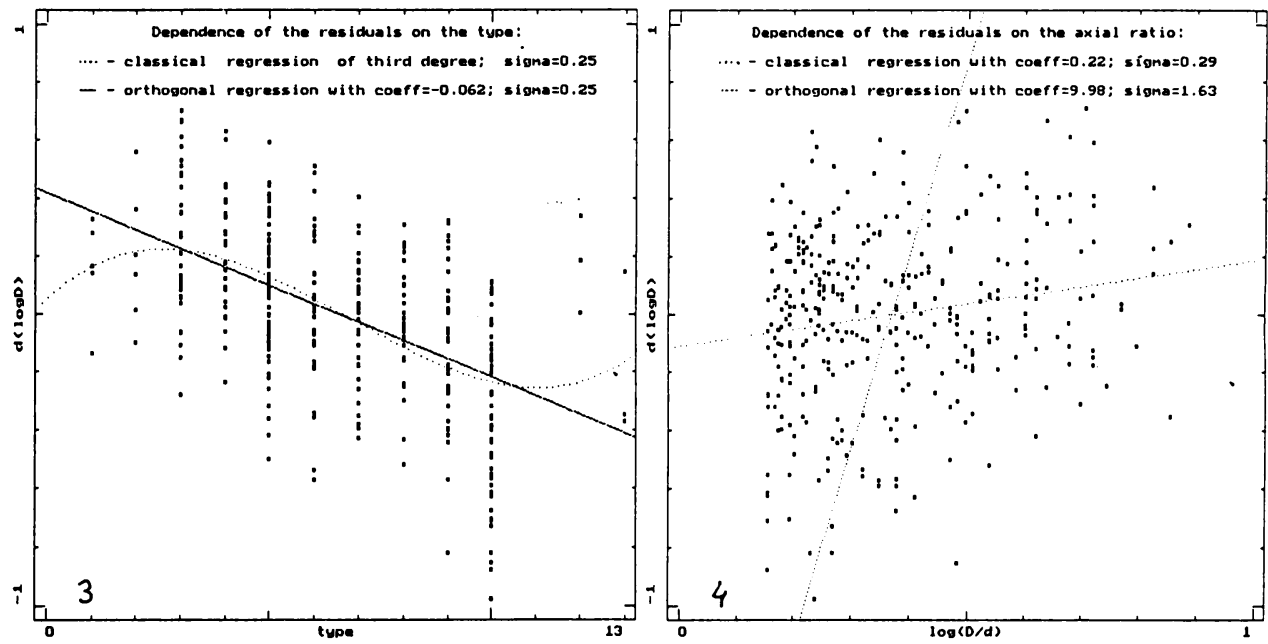

Figures 3 and 4. Dependence of the deviations from the deceleration law on the type and the axial ratio of the galaxy. 

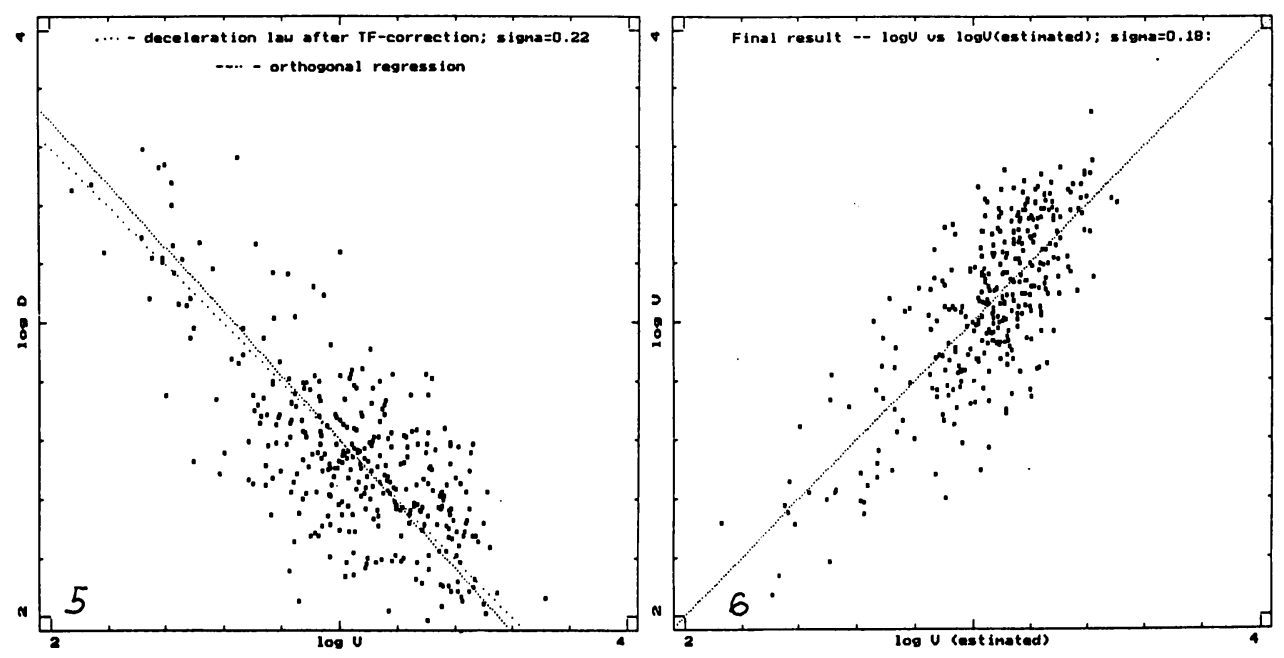

Figure 5. The deceleration diagram after TFcorrection which illustrates our unbiased performance of the TP-method; sigma $=0.22$.

Figure 6. Comparison between the observed velocities and the velocities obtained by the full multi-parametric method; sigma $=0.18$. 